\title{
Methods to Improve the Detection of Failures and Troubleshooting for Technical Diagnostics in Instrument
}

\author{
Kyaw Zaw Ye, Alexander M. Bain \\ Department of Computer Science and Software Computer Systems, National Research University of Electronic Technology (MIET), \\ Bld. 5, Pas. 4806, 124498, Zelenograd, Moscow, Russian Federation, \\ *Corresponding Author:kyawzawye85@gmail.com
}

Copyright (C) 2013 Horizon Research Publishing All rights reserved.

\begin{abstract}
This paper focuses on the development of techniques, which is based concept thinning of the flows together with the theory of regression analysis, proposed method of determining the expected number of failures to assess the efficiency of the technical diagnostics in instruments.
\end{abstract}

Keywords Technical Diagnostics, Failures, Troubleshooting, Identify, Instrument, Reliability

\section{Introduction}

At the present time, a number of high-tech industries, research and educational processes use many kinds of instrumentations which are greatly improve the efficiency of information processing. Despite the undoubtedly positive effect of the use of instruments, complex ongoing devices, we have to state their lack of effectiveness, due to a number of technical and economic circumstances. In particular, rather acute problem of improving the resiliency and reliability of elements of instrumentations, the life of which often exceeds the standard. In connection with the above, one of the most important requirements for instrument complexes is their high availability and the ability to effectively identify failures[1,2].

Theoretical and practical aspects of technical diagnostics, fault tolerance issues involved in instrument making such famous scientists as P.P Parkhomenko, Caribbean V.V, Sogomonyan E.S, Loaf M.F, A.V Lobanov, Schlichting R. , Rennels D.A, Dolev D. and many others etc.

The analysis showed that the modern industrial technologies used in various fields, require new approaches to ensure their reliability and effective methods of technical diagnostics. In this regard, there is a steady increase in the number of faults and failures, worsening the number of products increase the probability of accidents and crashes. Such a negative situation, which is related to the unreliability of the devices can be neutralized by increased personal skills, able to justify decisions, which is taken in the event of a negative situation.

\section{Methods of Determining the Expected Number of Failures in the Technical Diagnostics of Complex Instrumentation}

As noted in the introduction, one of the most important requirements for instrument complexes is their fault tolerance and the ability to effectively identify failures[3]. This paper proposes a mathematical description of the physical model of thinning of the flows in the annex to fault situations, and then the process of technical diagnostics (TD) can be formalized as follows.

There is an instrumental complex in which the design phase, "laid" fault situations (FS) of the total number of $\boldsymbol{T}_{f \boldsymbol{s}}$. During the $\boldsymbol{T D}$, they are identified, captured and eliminated. The recovery time during the $\boldsymbol{T D}$ is not taken into account. But $\boldsymbol{T D}$ continues at the end of the process of recovery.

The process of identifying the $\boldsymbol{F S}$ core flow of events failures. Each detected (shown itself) rejection reduces the intensity of the flow on the value of TFS with probability 1 , i.e., feed thins.

To develop a methodology for determining the expected number of failures apply the theory thinning flows, together with the regression analysis [4,5,6] under the following assumptions: the probability of detecting the FS is equal to 1, the probability of making a new FS in the recovery process is equal to 0 , a Poisson stream of refusals.

It is known that the thinning flows have functional characteristics which are successfully bind to parameters of statistics $\boldsymbol{T D}$ of devices and equipment of instrumentation.

Among such characteristics are the following:

Mathematical expectation (ME), number of failures will define to $(\mathrm{ME})$ :

$$
\overline{\mathrm{T}}_{\mathrm{fs}}\left(\mathrm{p}_{\mathrm{fs}}\right)=\mathrm{n}_{\mathrm{fs}}\left[1-\mathrm{e}^{-\mathrm{t}_{\mathrm{fs}} \mathrm{p}_{\mathrm{fs}}}\right] \text {; }
$$

Average value of the stream of refusals 


$$
\bar{t}_{f_{S}}\left(p_{f_{S}}\right)=n_{f_{s}} t_{f_{S}} e^{-t_{f_{s}} p_{f_{s}}}
$$

the ME time of total exhaustion failure flow

$$
\bar{P}\left(n_{f_{S}}\right)=\frac{1}{t_{f_{S}}} \sum_{\vartheta=1}^{n_{f_{s}}} \frac{1}{\vartheta}
$$

As well as the probability of not less than $\boldsymbol{T}_{\boldsymbol{f}}$, but it is equal to $\boldsymbol{T}_{f s}$ and $\boldsymbol{n}_{f s}$ exactly failures during number of $p_{f_{s_{i}}}$. They are respectively denoted $D_{f_{S}}\left(\mathrm{p}_{f_{S}}\right), D_{f_{S}}\left(\mathrm{p}_{f_{S}}\right)^{i}$, Last chance essence of the like lihood of complete exhaustion failure flow over time $\boldsymbol{P}_{\boldsymbol{f}}$.

In formulas (1) - (3) the following designations: $\boldsymbol{n}_{f s}$ - total (initial) number of $\boldsymbol{F S}$, embedded in the devices and equipment of instrumentation; $\boldsymbol{t}_{f s}$ - the instantaneous failure rate of thinning flow; $\vartheta-$ the current variable. Based on the experimental data one can determine the number $\boldsymbol{t}_{f s}$ and $\boldsymbol{n}_{f s}$ and other characteristics thinning flow are generated by the technical appliance diagnosis unit (apparatus) or the multi-computer system as a whole.

Below is the technique of determining, the expected number of failures on the basis of simple probabilistic methods using empirical data for practical purposes instrumentation. On the observed time interval $\left[0, \boldsymbol{P}_{\boldsymbol{f s}}\right]$ is $\boldsymbol{T}_{\boldsymbol{f s}}$ failures. The expectation value, by definition:

$$
M(\xi)=\int_{-\infty}^{\infty} X_{f_{s}} d F\left(X_{f_{s}}\right)
$$

where $\mathrm{F}$ - distribution function of the random variable .

$$
F\left(X_{f_{S}}\right)=\frac{1-e^{-t_{f_{S}} X_{f_{S}}}}{1-e^{-t_{f_{s}} p_{f_{S}}}}, \quad X_{f_{S}} \leq p_{f_{S}}
$$

The function $F\left(X_{f s}\right)$ takes the value 1 for $X_{f s}=p_{f s}$, ie All events take place later in point of the time $\boldsymbol{P}_{\boldsymbol{f} \text { s }}$. We rewrite the integral (4) with the normalizing $\frac{1}{1-e^{-t_{f s} p_{f s}}}$ factor that rightfully regarded as the events that occurred before.

$$
\begin{gathered}
M(\xi)=\int_{-\infty}^{\infty} X_{f s} d F\left(X_{f s}\right)=\frac{1}{1-e^{-t_{f s} p_{f s}}} \int_{0}^{p_{f s}} X_{f s} d\left(1-e^{-t_{f s} X_{f s}}\right) ; ;(6) \\
\text { Expression } \int_{0}^{p_{f s}} X_{f S} d\left(1-e^{-t_{f s} X_{f s}}\right) \text { integrate by }
\end{gathered}
$$

parts:

$$
\int_{0}^{p_{f s}} X_{f s} d\left(1-e^{-t_{f s} X_{f s}}\right)=-\int_{0}^{p_{f s}} X_{f s} d e^{-t_{f s} X_{f s}}=-\left.X_{f s} e^{-t_{f s} X_{f s}}\right|_{f_{f s}} ^{p_{f s}}+P_{t}\left(\eta=T_{f s} / \xi=n_{f s}\right)=\left(\begin{array}{c}
n_{f s} \\
T_{f s}
\end{array}\right)\left(1-e^{-t_{f s} p_{f s}}\right)^{T_{f s}} e^{-t_{f s} p_{f s}\left(n_{f s}-T_{f s}\right)}=
$$

$$
\begin{gathered}
\int_{0}^{p_{f_{s}}} e^{-t_{f_{s}} X_{f_{s}}} d X_{f_{s}}=-t_{f_{s}} e^{-t_{f_{s}} X_{f_{s}}}-\frac{1}{t_{f_{s}}} e^{-t_{f_{s}} p_{f_{s}}} \\
+\frac{1}{t_{f_{s}}}=\left(\frac{1}{t_{f_{s}}}-\frac{e^{-t_{f_{s}} p_{f_{s}}}}{t_{f_{s}}}\left(1+t_{f_{s}} p_{f_{s}}\right)\right)
\end{gathered}
$$

Then the equation (7) has the form:

$$
\frac{1}{1-e^{-t_{f s} p_{f s}}} \int_{0}^{p_{f s}} X_{f s} d\left(1-e^{-t_{f s} X_{f s}}\right)=\frac{\frac{1}{t_{f s}}-\frac{e^{-t_{f s} p_{f s}\left(1+t_{f s} p_{f s}\right)}}{t_{f s}}}{1-e^{-t_{f s} p_{f s}}} ;
$$

Rearranging equation (8),

$$
m_{f_{s}}=\frac{1-e^{-t_{f s} p_{f_{s}}}\left(1+t_{f_{s}} p_{f_{S}}\right)}{t_{f_{S}}\left(1-e^{-t_{f s} p_{f_{s}}}\right)}
$$

Transcendental equation, provided that a sufficiently large work tfs pfs can be neglected by $\left(1+t_{f_{s}} p_{f_{S}}\right)$ in the numerator (9) and the denominator is in the expression $e^{-t_{f_{s}} p_{f_{s}}}$. In the case of infinitesimal above $t_{f_{S}} p_{f_{S}}$ expressions can be also ignored. Then we have:

$$
m_{f s} \approx \frac{1}{t_{f_{S}}}
$$

The value $\boldsymbol{m}_{\boldsymbol{f}}$ is biased estimate for the entire set of events, but in the interval $\left[0, p_{f_{S}}\right]$, it is not biased and is the sum of $\boldsymbol{T}_{\boldsymbol{f s}}$ events (failures) at times $p_{f_{s}}$. The expected number of failures is defined as follows:

$$
\begin{aligned}
& m_{f_{s}}=\frac{1}{T_{f_{s}}} \sum_{i=1}^{T_{f_{s}}} p_{f_{s}} ; \\
& t_{f_{s}}=\frac{T_{f_{s}}}{\sum_{f_{s}} p_{f_{s}}} .
\end{aligned}
$$

The expected number of failures is defined as follows.

Then: $\xi=n_{f_{S}}$ 


$$
\begin{gathered}
\left(1-e^{-t_{f s} p_{f s}}\right)^{T_{f s}} e^{t_{f s} p_{f s} T_{f s}} \sum_{n_{f s}=T_{f s}}^{\infty}\left(\begin{array}{c}
n_{f_{s}} \\
T_{f s}
\end{array}\right) e^{-t_{f s} p_{f s} n_{f s}}= \\
=\left(1-e^{-t_{f s} p_{f s}}\right)^{T_{f s}} e^{t_{f_{s}} p_{f_{s}} T_{f_{s}}} \\
\sum_{n_{f s}=T_{f s}} \frac{n_{f_{s}}\left(n_{f s}-1\right) \ldots\left(n_{f s}-T_{f_{s}}+1\right)}{T_{f s}} e^{-t_{f s} p_{f s} n_{f s}}
\end{gathered}
$$

To simplify the calculations, we introduce the following notation.

$e^{-t_{f_{s}} p_{f_{s}}}=X_{n w}$. Then,

$$
\begin{gathered}
\sum_{n_{f s}=T_{f s}}^{\infty}=\frac{X_{n w}{ }^{T_{f s}} T_{f s} !}{\left(1-X_{n w}\right)^{T_{f s}+1}}= \\
T_{f s} ! \times\left[X_{n w}{ }^{T_{f s}}\left(1-X_{n w}\right)^{-T_{f s}-1}\right]= \\
T_{f s} !\left[\mathrm{T}_{f s} X_{n w}{ }^{T_{f s}-1}\left(1-X_{n w}\right)^{-T_{f s}-1}-\right. \\
-\left(\mathrm{T}_{f s}+{ }_{f s}\right)^{T_{f s}}\left(\frac{1}{1-X_{n w}} X_{n w}{ }^{T_{f s}}\left(1-X_{n w}\right)^{-T_{f s}-2}\right]= \\
\frac{T_{f s} !\left\{X_{n w}{ }^{T_{f s}-1}\left(1-X_{n w}\right)^{-T_{f s}-2}\left[\mathrm{~T}_{f s}\left(1-X_{n w}\right)-\left(\mathrm{T}_{f s}+1\right) X_{n w}\right]\right\}=}{\left(1-X_{n w}\right)^{T_{f s}+1} T_{f s} ! X_{n w}{ }^{T_{f s}}\left(\mathrm{~T}_{f s}-2 T_{f s} X_{n w}-X_{n w}\right)}=\frac{1-X_{n w}}{T_{f s}-2 T_{f s} X_{n w} .}
\end{gathered}
$$

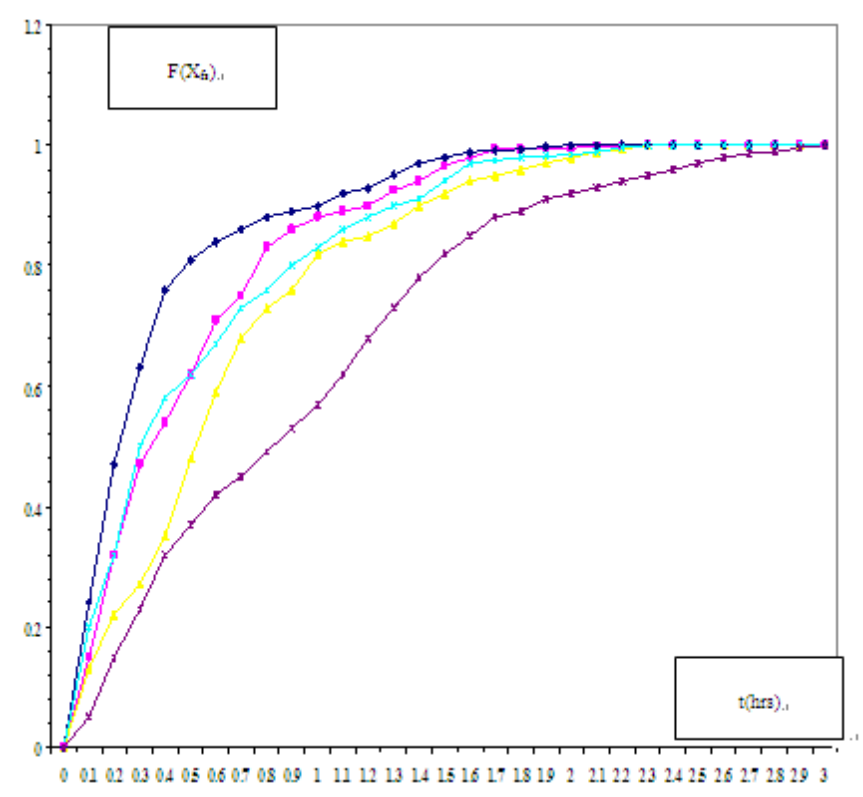

Figure 1. The empirical dependence of the failure distribution 
Figure 1 shows the empirical dependence of the distribution function of the number of failures over time. The value of the failure rate tfs find from equation (10) by defining the value of an empirically constructed by the tabular method of curve and normalized distribution function: a time $p_{f_{S}}=t_{1}$ failures occurred $\mathrm{n}_{1}$, through $p_{f_{S}}=t_{1}$ failures occurred $\mathrm{n}_{2}$, etc.

The results lead to the following conclusions. First, we can estimate the efficiency of TD by analyzing the dependence of $T\left(n_{f s}\right)=f\left(n_{f s}, t_{f s}\right)$.

If the time TD $\sim \boldsymbol{t}\left(\boldsymbol{n}_{f}\right)$, the quality (effectiveness) diagnosis can be regarded as acceptable. If $T\left(n_{f_{S}}\right)>>$ $p_{f_{S}}$, on the program of sufficient "hard", the intensity of the flow of failure is low. In this case, you should review and revise the program of the AP. If a large number of $n_{f s}$, it is necessary to review and revise the production technology products. The function $T\left(n_{f_{S}}\right)=f\left(n_{f_{S}}, b_{f_{S}}\right)$ does not have an unconditional extreme inside the interval of existence. Constrained optimization can be found, if there are known limitations.

Second, the model adopted and the results of statistical data processing TD can solve two "side", but is essential to the practice of the problem

\section{Methods of Detection of Single Failures in the Automated System of Technical Diagnosis}

Automation system of technical diagnostics (ASTD) belongs to a class of large technical systems, consisting of a large number of components and elements with a complex structure. Tasks diagnostics (detection and retrieval failures) of such systems is very difficult, so ASTD should be viewed as an object of technological diagnostics (OTD). Then the system can be represented as a set of $\mathrm{n}$ of its constituent elements (set), connected by a functional linkages [4].

The probability of working condition of the item is indicated $p_{i}^{o t i}$, and the probability of failure $q_{i}^{o t i},\left(q_{i}^{o t i}\right.$ $\left.=1-p_{i}^{o t i}\right)$. It is assumed that the failures of the system components mutually independent.

Health monitoring systems is the use of special tests, and each of which checks are well-defined to subset of elements. The check is made for the following purposes:

- check the system (detecting any failure of existing);

- refusal to find (search all failed elements).

In the first case, it suffices to apply a test that checks the whole system (the so-called general test). However, sometimes conduct such a test is fundamentally impossible, or inexpedient, since it requires considerable time and (or) it means the advantageous to use an aggregate of a few simple tests.
To the monitor performance and looking for places bounce OTD has a possibility of testing, $h_{i}^{\text {oti }}, i=1, \ldots$, $m^{o t i}$, allowing check $m^{\text {oti }}$ parameters, the nominal value of each of which is provided by a subset of functional elements $\Omega_{i}$. The test may consist of supplying the necessary input to measure the response in one or more control points, etc. The results of the application of each test are classified on a "successful" if all the elements of workable $\Omega$ and "not successful" if refused by at least one member of $\Omega_{i}$. The application of each test involves some costs $c_{i}^{\text {oti }}$, which may indicate the time or cost required to verify the parameter value required for this equipment, etc. The set of tests is more convenient to define a matrix, $H^{o t i}=\left|h_{i j}^{o t i}\right|, i=$ $1, \ldots, m^{o t i}, j=1, \ldots, n^{o t i}$, lines which swarm match the available tests, and columns - elements of the set. Thus,

$$
h_{i j}^{o t i}= \begin{cases}1, & \text { прu } j \in \Omega_{i} \\ 0, & \text { npu } j \in \Omega_{i} \quad i=1, . ., m^{\text {oti }}, 1 \ldots, n^{\text {oti }} .\end{cases}
$$

Column vector of coti $=\left\{\right.$ ci oti, ..., $\left.\quad c_{m^{o t i}}^{o t i}\right\}$ determines the cost associated with the use of each test. We assume that for the task of control ASTD existing tests enough, we now define the procedure on the basis of their current classification .

Control processes are classified by a number of attributes:

A. In depth, fault localization distinguishes control processes of the working capacity of the system as a whole and to determine the diagnosis of the state of each element.

B. According to the method of the control procedures can be divided into a sequence and combination. In the first case the choice of the next test is performed with a conventional program which is based on analysis of previous audits. In the second case, the true state of OTI is determined after the application of the entire set of tests.

C. Serial control procedures are usually evaluated in two optimality criteria: minimum average cost of the program and the minimum of the maximum value of this quantity.

D. With an a priori definition of the set of admissible states OTD are usually in to applied two hypotheses : OTD is likely to fail in not more than one member; Various combinations of failed components at the same time.

E. If it is allowed to arbitrary combinations of simultaneous failures, the sequence of monitoring procedures can be combined with the recovery of failed elements as they are discovered or occur without recovery. In the first case is up to full OTD, in the second to - set the status of each element, i.e., 
to identify the state of OTD.

F. From the combination of elements that remain untested after control, distinguishes to control with complete and incomplete coverage of elements of OTD.

G. By the degree of reliability of an inspection instrumentation distinguish reliable and unreliable control.

H. According to the degree of detail of the information is about the state of OTI, which is received as a result of monitoring distinguish decision-making problem about the true state of OTI in complete and incomplete information [2].

We formalize the task to identify the defective item. It is known for a exact failure of a OTD, given a matrix of tests $H^{o t i}$ and the probability of failure of each element, $q_{i}^{o t i}, i=$ $1, \ldots, n^{o t i}$. Need a certain group of selected test sufficient for finding the failed element and define the order of sequential application of a conditional test of this group (program) so that the average value of the total cost of the search procedure to a minimum.

The use of any test $h_{i}^{\text {oti }}$ can be regarded as a partition of the elements $\Omega$ into two subsets: $\Omega_{i}$ and $\overline{\Omega_{i}}$. At the end of "not successful" failed element is in the subset $\Omega$, at the end of "successful" in a subset $\overline{\Omega_{i}}$.

For further localization of failure tests can be used $h_{\mathrm{i}} \in H^{\text {oti }}$ (here in after referred to as essential), allowing to carry out further divide the subsets $\Omega_{i}$ or $\overline{\Omega_{i}}$, containing the failed element. Test $\mathrm{h}_{\mathrm{i}}$ is essential for a subset $\Omega_{i}$, if:

$$
\left\{\begin{array}{l}
\Omega u \cap \Omega_{i} \neq \varnothing \\
\Omega u \cap \Omega_{i} \neq \Omega_{i}
\end{array} .\right.
$$

The list of essential tests for $\Omega_{i}$, denote $H_{i}$, and if the two tests and $\mathrm{h}_{\mathrm{i}}$ and $\mathrm{h}_{\mathrm{v}} \Omega_{\mathrm{i}} \bigcap \Omega_{i}=\Omega_{v} \bigcap \Omega_{i}$, then the list $H_{l}^{\text {oti }}$ leave one, which correspond to lower costs.

List $H_{l}^{\text {oti }}$ can be a matrix whose columns correspond to the elements $\Omega_{i}$. Each k-th row of the matrix corresponds to a test of $\boldsymbol{h}_{\boldsymbol{u}(\boldsymbol{i})}^{k}, k=1, \ldots, m_{i}^{\text {oti }}$, monitors the health of subsets $\Omega_{u(i)}^{k}=\Omega_{\mathrm{u}} \bigcap \Omega_{i}$ and at a cost $Z_{u(i)}^{k}=Z_{u}$. Subscript without parentheses is used to identify each test in the original matrix $H^{\text {oti }}$. Sometimes, for simplicity it will be omitted. Similarly, it can be formed in a matrix of tests that are essential for $\overline{\Omega_{i}}$, if further search failed element is carried out in this subset.

If OTD is only one possible failure, the sign of the adequacy of the matrix $H^{\text {oti }}$ is containment of any failure for the level of the element which is that all on the columns of the matrix. They must be pair wisely different [6].

\section{Conclusion}

1. Confirm the reliability of the product individual parameter. Indeed, if the statistics are processed TI single (one) product, $p_{f_{S}}=T\left(n_{f_{S}}\right)$ then the flow of failures can assume virtually stationary and therefore, $t_{f s}\left[t_{f s} \geq T\left(n_{f s}\right)\right]=\lambda_{f s}$.

2. The plan is reasonably for plan warranty products. Knowing the expected number of fault situation, "embedded" in the product, the number of detected faults, and the probability of failures at certain intervals of time, can determine the amount of the stock of tools and accessories (spare parts), the number and size of repair crews, timelines (stochastic) of their operations, financing activities, etc.

3. The methods of detection of single failures during the technical diagnostics aimed at a variety of tests, sufficient for finding the failed component, and the definition of a conditional order of the consistent application of tests on the criterion of minimizing the average total cost of the search.

\section{REFERENCES}

[1] Kyaw Zaw YE, Kukushkin E.S, Lisov O.I. Fault Finding in the multi-computer computer systems based on the "AND-OR" graph / / journal of "The defense complex - the scientific and technical progress of Russia." -M.: Federal State Unitary Enterprise "VIMI," 2012. - № 1. - C.71-75

[2] Kyaw Zaw Ye. Method for determining a single fault with the technical diagnosis of multiple computer systems / / Scientific and practical journal "Industry aspects of Engineering." - M.: Publishing INGN, 2012. - № 1. - C.18-19.

[3] Kyaw Zaw Ye. Evaluation model of CPU performance of distributed fault-tolerant automation systems / / Modern information technology. International Scientific and Technical Conference: Collected statey., Penza, 2013. C.124-127.

[4] Kazak D.S. The model for the evaluation of fault tolerance of automated control devices and diagnostics / / Proceedings of the universities. Electronic.-M.: MIET, 2009. - № 1.-C.89-92.

[5] Kazak D.S. A mathematical model of CPU performance evaluation of distributed automation systems for process testing / / Scientific and technical journal "Engineering and Technology", Moscow: Publishing House of the "Company Sputnik." - 2008, № 5.-C.37-38.

[6] Kazak D.S .The checks performance of automated systems for technological tests in instrument / / Scientific and technical journal "Engineering and Technology", Moscow: Publishing House of the "Company Sputnik." - 2008, № 5.-C.39-40. 